\title{
Excessive Dpp signaling induces cardial apoptosis through dTAK1 and dJNK during late embryogenesis of Drosophila
}

\author{
Sheng-An Yang and Ming-Tsan Su
}

\begin{abstract}
Background: To identify genes involved in the heart development of Drosophila, we found that embryos lacking raw function exhibited cardial phenotypes. raw was initially identified as a dorsal open group gene. The dorsal open phenotype was demonstrated to be resulted from the aberrant expression of decapentaplegic (dpp), a member of the tumor growth factor beta (TGF- $\beta$ ), signaling pathway. Despite the role of $d p p$ in pattering cardioblasts during early embryogenesis of Drosophila have been demonstrated, how mutation in raw and/or excessive $d p p$ signaling involves in the differentiating heart of Drosophila has not been fully elaborated at late stages.

Results: We show that raw mutation produced a mild overspecification of cardial cells at stage 14, but these overproduced cells were mostly eliminated in late mutant embryos due to apoptosis. Aberrant $d p p$ signaling is likely to contribute to the cardial phenotype found in raw mutants, because expression of $d p p$ or constitutively activated thickven $\left(t k v{ }^{C A}\right)$, the type I receptor of Dpp, induced a raw-like phenotype. Additionally, we show that $d p p$ induced non-autonomous apoptosis through TGF $\beta$ activated kinase 1 (TAK1), because mis-expression of a dominant negative form of Drosophila TAK1 ( $d T A K 1^{D N}$ ) was able to suppress cell death in raw mutants or embryos overexpressing dpp. Importantly, we demonstrated that dpp induce its own expression through dTAK1, which also leads to the hyperactivation of Drosophila JNK (DJNK). The hyperactivated DJNK was attributed to be the cause of Dpp/DTAK1-induced apoptosis because overexpression of a dominant negative DJNK, basket (bsk ${ }^{\text {DN }}$ ), suppressed cell death induced by Dpp or DTAK1. Moreover, targeted overexpression of the anti-apoptotic P35 protein, or a dominant negative proapoptotic P53 (P53 ${ }^{\mathrm{DN}}$ ) protein blocked Dpp/DTAK1-induced apoptosis, and rescued heart cells under the raw mutation background.

Conclusions: We find that ectopic Dpp led to DJNK-dependent cardial apoptosis through the non-canonical TGF- $\beta$ pathway during late embryogenesis of Drosophila. This certainly will increase our understanding of the pathogenesis of cardiomyopathy, because haemodynamic overload can up-regulate TGF- $\beta$ and death of cardiomyocytes is observed in virtually every myocardial disease. Thus, our study may provide possible medical intervention for human cardiomyopathy.
\end{abstract}

\section{Background}

The Drosophila heart is a simple tubular organ located at the dorsal midline beneath the epidermis, and it is therefore alternatively termed the dorsal vessel. The fly heart consists of two major cell types, myocardial cells and pericardial cells, which arise from two bilateral rows of cardiac primordia at the leading edge of the migrating

\footnotetext{
* Correspondence: mtsu@ntnu.edu.tw
Department of Life Science, National Taiwan Normal University, Taipei 11677,

* Correspondence: mtsu@ntnu.edu.tw
Department of Life Science, National Taiwan Normal University, Taipei 11677, Taiwan
}

(c) 2011 Yang and Su; licensee BioMed Central Ltd. This is an Open Access article distributed under the terms of the Creative Commons Attribution License (http://creativecommons.org/licenses/by/2.0), which permits unrestricted use, distribution, and reproduction in any medium, provided the original work is properly cited.

mesoderm. The contractile myocardial cells which form the lumen are arranged in a segmental repeat comprised of six cells per hemisegment in the mature embryonic heart. The pericardial cells, which are essential for normal cardiac function, are aligned alongside the myocardial cells. Despite its simple structure, fly heart has recently emerged as an excellent model system for dissecting the complex pathway that determines cardiogenic cell fate, and for investigating the physiologic function of the adult heart $[1,2]$. 
Extensive study has revealed that a combinatory action of extrinsic signaling and intrinsic transcription network is required for correct specification of cardial precursors and differentiation of mature heart (reviewed in [3]). Of all external signalings, Dpp, a member of the mammalian Transforming growth factor superfamily (TGF- $\beta$ ), has been shown to play a pivotal role during cardiogenesis of Drosophila [4]. The cardiogenic function of Dpp begins when it is expressed in the dorsal epidermis in a broad band along the anterior-posterior axis during germ band extension in Drosophila [5]. This spatiotemporal pattern of Dpp specifies the underling dorsal mesodermal cell fate by maintaining the expression of the transcription factor, tinman (tin) [4,6-8]. Dpp also regulates the expression of several other cardiogenic transcription factors, including pannier (pnr) and dorsocross $(d o c)[9,10]$. For further specification of the cardiogenic mesoderm, Wg signaling together with the combinatorial action of several transcription factors, including tin, pnr, doc and tailup, are required [11-20]. Around stage 10, Dpp expression in the dorsal ectoderm vanishes briefly, but reappears in the leading edge (LE) cells of the dorsal ectoderm at stage 11. This second round of Dpp expression in LE cells persists through stage 17 [21]. Interestingly, pMad, the activated Dpp signal transducer, can be detected in a subset of cardial progenitors in stages 12 to 14 [22]. This indicates that a second round of Dpp activity is required for further differentiation of Drosophila heart. Indeed, $d p p$ mutants with alleles that affect the expression of Dpp in LE cells have impaired embryonic heart development and larval cardiac function $[23,24]$. These findings indicate a biphasic requirement for Dpp during cardiogenesis of Drosophila, in which it is required early for dorsal mesoderm patterning and later for differentiating heart cells.

Dpp regulates many developmental processes, including cell fate determination, alteration of cell shape, proliferation, and apoptosis. Morphogenic function of Dpp in cell fate determination has been shown to be mediated through the canonical pathway, in which it interacts with a type I receptor, Tkv, and a type II receptor, Punt. Upon formation of ligand-receptor complex, activated Punt phosphorylates Mad, which subsequently interacts with Medea. The resultant complex containing pMad, and Medea is then translocated into the nucleus where it activates transcription of Dpp target genes [25]. Other than the canonical pathway, it has been found that mammalian Dpp homolog, TGF- $\beta$ transduce its signaling that is independent of Smad, a homolog of Drosophila pMad. The Smad-independent pathway is designated as the non-canonical TGF- $\beta$ pathway. In the non-canonical pathway, TGF- $\beta$ activated kinase 1 (TAK1) forms a multiple protein complex protein complex with TRAF6, TAB2, and TAB3. Upon binding of TGF- $\beta$ to its receptor, TRAF6 exerts its E3 ubiquitin ligase activity together with ubiquitin-conjugating enzymes to catalyze Lys63-linked polyubiquitination. Subsequently, the Lys63-linked polyubiquitin chain associates with TAB2 which leads to autophosphorylation and activation of TAK1 [26,27]. Despite it is less clear in Drosophila, many functionally-conserved non-canonical signaling transducers, including DTAK1 and TAB2, have been identified in Drosophila [28,29]. Moreover, Dpp signaling has also been shown to control the viability of cells. A lack of Dpp signaling activates c-Jun N-terminal kinase (JNK)-dependent apoptosis in wing discs [30]. Interestingly, Dpp is also likely to function as a pro-apoptotic signal because increased Dpp activity leads to both non-autonomous JNK activation and cell death [31]. However, the link between Dpp signaling and JNK-mediated apoptosis is currently unclear in Drosophila.

Dpp is a downstream target of the JNK pathway, a conserved and pleiotropic signaling system whose function governs many different biological activities, including morphogenesis, differentiation, proliferation and apoptosis. Components of the DJNK pathway, including Djun and Raw, have been shown to participate in Drosophila heart development by modulating the expression of Dpp $[14,16]$. In Djun mutant embryos, the expression of $d p p$ is not maintained at dorsal edge, which leads to downregulation of cardiac tin at later stages [16]. By contrast, pericardial cells are overspecified in raw loss-of-function mutant embryos [14]. The excessive differentiation of cardial cells has been attributed to the ectopic Dpp activity induced by dysregulated DJNK signaling.

In our effort to identify genes involved in the heart development of Drosophila, we observed that embryos lacking raw function exhibited cardial phenotypes, in which heart cells were overspecified in moderately degree during mid-embryogenesis, and that the overproduced heart cells had disappeared at late stages. We show here that the elimination of heart cells in late raw mutant embryos was a result of excessive apoptosis, and ectopic Dpp signaling was responsible for the cardial apoptosis phenotypes of raw mutant embryos. We also found that elevated Dpp can function as a pro-apoptotic signal to promote non-autonomous apoptosis in a dose-dependent manner. Importantly, ectopic Dpp auto-regulate its own expression through DTAK1. The autocrine Dpp further enhanced the expression of DJNK and consequently led to P53-dependent cell death. Our study defined a novel pathway which linked ectopic Dpp signaling and DJNK-dependent apoptosis during late cardiogenesis of Drosophila.

\section{Methods}

\section{Fly stocks and genetics}

Fly stocks were raised and crossed at $25^{\circ} \mathrm{C}$. Gal4 drivers: Act5C-gal4 (constitutive), 24B-gal4 (mesodermal), and 
$69 B$-gal4 (ectodermal) were obtained from the Bloomington Stock Center. The $s v p$ specific enhancer trap line E2-3-9 has been described [32]. him-GFP, a reporter in which a $2.2 \mathrm{~kb}$ genomic fragment spanning the promoter region and 5' untranslated region of him was placed upstream of nuclear green fluorescent protein encoded gene reporter, was used to mark the muscle, cardial and pericardial cells [33], The $d p p$-LacZ BS3.0 reporter reflects the transcription of $d p p$ in embryos [34]. $r a w^{1}$ has been described [35]. UAS- $d p p$, UAS- $b s k$, UAS- $b s k^{D N}$, UAS- $p 35$ [36]; UAS- $p 53$, UAS- $p 53^{D N}$, UAS$b r k, t k v^{7}$ and $t k v^{C A}$ were also obtained from the Bloomington Stock Center. UAS-tkv-RNAi (102319, obtained from the Vienna Drosophila RNAi Center, VDRC); UAS- $d T A K 1$ and UAS- $d T A K 1^{D N}$ [29] were gifts from $\mathrm{T}$. Adachi-Yamada. The isogenic $w^{1118}$ flies were used as wild-type (WT) controls.

\section{Plasmid construction and generation of transgenic flies}

The UAS/Gal4 binary expression system was used to drive the expression of transgenic constructs [37]. For generation of the UAS-raw transgenic construct, the full-length raw cDNA was amplified using pNB3301 (obtained from A. Letsou) as template with a pair of primers (5'-CACCATGAAAACTGAAAGCAGCAGT-3' and 5'-GCAGC GGTCGCGGTTGTTGT-3') by PCR. The amplified DNA fragment was first cloned into a pENTR/D-TOPO vector (Invitrogen), and subsequently into a pTWF vector (The Drosophila Genomics Resource Center). The plasmid construct was confirmed by sequencing before germ-line transformation. The raw-RNA interference construct, UAS-raw-RNAi, was generated by amplification of a 693 bp DNA fragment using the following primers, 5'GCTCTAGACCTGGAGCGCCAGAGTCTC-3' and 5'GCTCTAGATGACGAAGAGCAACACTCG-3'. The amplified DNA fragment was digested with $\mathrm{XbaI}$ and cloned into the $A v r I I$ site of a pWIZ vector. The correct clone was used to clone the same XbaI-digested DNA fragment into the NheI site, as described elsewhere [38]. The orientation of the inverted DNA fragment was confirmed by restriction enzyme digestion. Flies carrying transgenic constructs were generated by $P$-elementmediated germ-line transformation procedure using $w^{1118}$ as the parental line [39].

\section{RT-PCR}

Total RNA was purified from embryos carrying rawRNAi transgene driven by Act5C-gal4 driver using Trizol (Invitrogen). $10 \mu \mathrm{g}$ of total RNA was reverse transcribed using oligo(dT) primers and SuperScript reverse transcriptase (Invitrogen). PCR was performed using raw specific primers (5'-TACCATAAGCACCGCCAGCA-3' and 5'-ATGCGAACTGGCCGAGGATC-3') and rps17 primers (5'-CGAACCAAGACGGTGA
AGAAG-3' and 5'-CC TGCAACTTGATGGAGATACC-3'). PCR condition: at $94^{\circ} \mathrm{C}$ for $2 \mathrm{~min}, 30$ cycles (at $94^{\circ} \mathrm{C}$ for $30 \mathrm{sec}$, at $46^{\circ} \mathrm{C}$ for $30 \mathrm{sec}$, at $72^{\circ} \mathrm{C}$ for 1 $\min )$, and at $72^{\circ} \mathrm{C}$ for $7 \mathrm{~min}$.

\section{Cell death detection}

Apoptosis was detected by acridine orange (AO) staining or terminal deoxynucleotidyl transferase-mediated dUTP nick end labeling (TUNEL). For AO staining, the same protocol was followed as used elsewhere [40]. Briefly, dechorionated embryos were stained by placing in an equal volume of $n$-heptane and $1 \times$ PBS containing $5 \mathrm{mg} / \mathrm{ml}$ of acridine orange (Sigma) for 5 minutes on a shaking platform. For TUNEL analysis, an in situ cell death kit was used according to the manufacturer's instructions (Roche Applied Science). To detect cardiac apoptosis in embryos, a TUNEL and immunoflourescence double-labeling protocol was followed as described [41]. Stained embryos were mounted either with mineral oil (Sigma) or series 700 Halocarbon oil (Halocarbon Products, Hackensack, NJ). Samples were viewed either with a fluorescence or TCS SP2 confocol microscope (Leica Microsystems).

\section{Immunohiscytochemistry, X-gal staining and cuticle preparation}

Immunohistochemical staining was performed as described [42]. Antibodies and dilutions were as follows: Anti-Eve, anti-Wg 4D4 and anti-EC11 (1:10; obtained from the Developmental Studies Hybridoma Bank), antiTin (1:2000, provided by M. Frasch), anti-LacZ, antiGFP (1:200, Molecular Probes), and anti-pMad (1:20, Cell Signaling) [43]. Appropriate anti-mouse or anti-rabbit HRP-conjugated secondary antibodies were used at a dilution of 1:200 (Jackson ImmunoResearch). The staining pattern was visualized using the Vectastain $A B C$ kit (Vector Laboratories). X-gal staining was performed as described previously with slight modifications [44]. Briefly, collected embryos were dechorionated in $50 \%$ bleach for $90 \mathrm{~s}$ and fixed in 4\% formaldehyde buffered with $1 \times$ PBS for $20 \mathrm{~min}$. Fixed embryos were washed briefly and incubated with $\mathrm{X}$-gal staining solution (10 $\mathrm{mM}$ sodium phosphate, $\mathrm{pH} 7.2,150 \mathrm{mM} \mathrm{NaCl}, 1 \mathrm{mM}$ $\mathrm{MgCl}_{2}, 3 \mathrm{mM} \mathrm{K}_{4}\left[\mathrm{Fe}(\mathrm{CN})_{6}\right], 3 \mathrm{mM} \mathrm{K}_{3}\left[\mathrm{Fe}(\mathrm{CN})_{6}\right], 0.3 \%$ Triton $\mathrm{X}-100,0.2 \% \mathrm{X}$-gal) at $25^{\circ} \mathrm{C}$. Immuno- and $\mathrm{X}$-gal stained embryos were mounted in $50 \%$ glycerol. For preparation of cuticle, embryos were fixed in glycerol-acetic acid (1:4) and cleared in Hoyer's medium overnight at $60^{\circ} \mathrm{C}$ [45]. Stained embryos and cuticle were visualized with a light microscope (Leica DMR A2).

\section{Image acquisition and processing}

Epifluorescence images were acquired using a digital camera (CoolSnap 5.0, Photometrics) steered by the 
Northern Eclipse 6.0 software (EMPIX Imaging, Mississauga, Ontario, Canada). When necessary, Z-series of optical or fluorescent images were acquired at $2 \mu \mathrm{m}$ increments with a piezo-electric motor (LVDT, Physik Instruments). The Helicon Focus program was applied to combine the focused images (Helicon Soft Ltd. Kharkov, Ukraine). All the figures were arranged in Adobe Illustrator CS3 (Adobe Co.).

\section{Results}

\section{Loss of raw function impairs heart and muscle development}

In our effort to identify genes involved in the heart development of Drosophila, we found that many cardial cell types were missing in raw mutant embryos. For instance, using anti-Eve antibodies we found that Evenskipped positive pericardial cells (EPCs) were aligned normally along the dorsal vessel in wild-type embryos (Figure 1A). However, these EPCs almost completely disappeared in the raw mutant at stage 16 (Figure 1D). Similarly, absence of pericardial cells was observed in raw mutant using anti-EC11 and anti-Odd antibodies, which labelled extracellular matrix and Odd-skipped pericardial cells (OPCs), respectively, of pericardial cells (Figure 1B, E and Additional file 1, Fig. S1). Additionally, using a heart-specific enhancer trap line, E2-3-9, we found that $s v p$-expressing myocardial cells were reduced and/or missing under the raw mutant background (Figure $1 \mathrm{C}$ vs. $1 \mathrm{~F}$, arrow). To further investigate whether raw is involved in the early cardiogenesis of Drosophila, we used Tin as a marker because it is expressed initially in all cardial progenitors and later in four of six cardioblasts per hemisegment as well as a subset of pericardial cells in the mature embryonic heart (Figure 1H and 1I; see also [46-49]). We found that the expression of Tin in the dorsal mesoderm was normal in both wild-type and raw mutant embryos at stage 12 (Figure 1G and 1J). However, Tin-expressing heart cells were mildly overproduced in raw mutants at stage 14 (Figure $1 \mathrm{~K}$, braces). Nevertheless, these overproduced heart cells were reduced and/or absent in raw mutants during late embryogenesis (Figure 1L). Since the above markers label most of the cardial and pericardial cell types, our results suggest that the raw mutation affects all the cardial cell types in developing Drosophila heart. Based on the above observations, we thus concluded that heart cells were over-specified mindedly during mid-embryogenesis, and that these overproduced heart cells disappeared at late stages in raw mutant embryos.

To further confirm our findings, we used a him-GFP reporter in which a cardial-specific enhancer of him was placed upstream of nuclear GFP [33]. The GFP reporter was expressed in the precursors of both muscle and heart cells at stage 12 and its cardiac expression persisted till late embryogenesis in the differentiated heart cells under the control of tin, while its muscle expression was greatly reduced at stage 14 (Figure $1 \mathrm{M}$ and $1 \mathrm{~N}$; and $\mathrm{M}-\mathrm{T}$. Su unpublished results). We found that the expression of him-GFP was increased in both heart and musculature at stage 14 under the raw mutation background (Figure 1P). Consistent with the above data, cardiac expression of him-GFP decreased significantly in mutant embryos at stage 16 (Figure 10 and $1 Q)$. Taken together, these data show that heart precursors are overspecified during mid-embryogenesis, but are missing in late mutant embryos.

\section{Down-regulation of raw causes cardial apoptosis}

How could the cardial cells disappear in the raw mutant embryos at later stages? One possibility is that loss of raw function induces programmed cell death (PCD). To investigate whether cardial apoptosis occurred in raw mutant embryos, we stained embryos with a vital dye, acridine orange $(\mathrm{AO})$, which provides a rapid visual assessment of apoptosis in live Drosophila [40]. Epifluorescence micrography showed apoptotic cells, mainly in the cephalic ganglia and in head regions in wild-type embryos at stage 14 (Figure 2A). A similar AO staining pattern was detected in $\mathrm{raw}^{1}$ mutant embryos at the same stage (Figure 2D). Excessive cell death in the dorsal mesoderm was observed in $\mathrm{raw}^{1}$ mutant embryos at stage 16 (Figure $2 \mathrm{~B}$ vs. 2E). To verify the above findings, terminal deoxynucleotidyl transferase dUTP nick end labeling (TUNEL) was applied. Indeed, TUNEL positive nuclei were identified in the dorsal mesoderm of $\mathrm{raw}^{1}$ mutant embryos at stage 16 (Figure $2 \mathrm{C}$ vs. $2 \mathrm{~F}$ ). We then made use of RNA interference to specifically knock down the expression of raw (Figure $2 \mathrm{G}$ and $2 \mathrm{H}$ ), and the results were consistent with the above findings, founding that silencing the expression of endogenous raw in ectoderm using 69B-gal4 successfully induced localized apoptosis in the dorsal mesoderm of embryos (Figure 2H).

Since the embryonic heart of Drosophila is located in the dorsal mesoderm, which was also the region where cell death was present in the raw mutants, we suspected that PCD is the cause of eliminating cardial cell types in the raw mutant. To test this, we double-labeled raw mutant embryos with TUNEL and heart-specific himGFP reporter (Figure 2I-L). By confocal microscopy analysis, the results showed that many him-GFP expressing cells were co-labeled with TUNEL, indicating that mesodermally-derived tissues did undergo apoptosis (Figure 2I, yellow nuclei surrounded with green cytoplasma). Since the expression of him-GFP was mainly in cardial and muscle cells under raw mutation background at late stages (Figure $1 \mathrm{Q}$ ), these results indicate that raw mutation does induce death of heart cells. Apart from the 


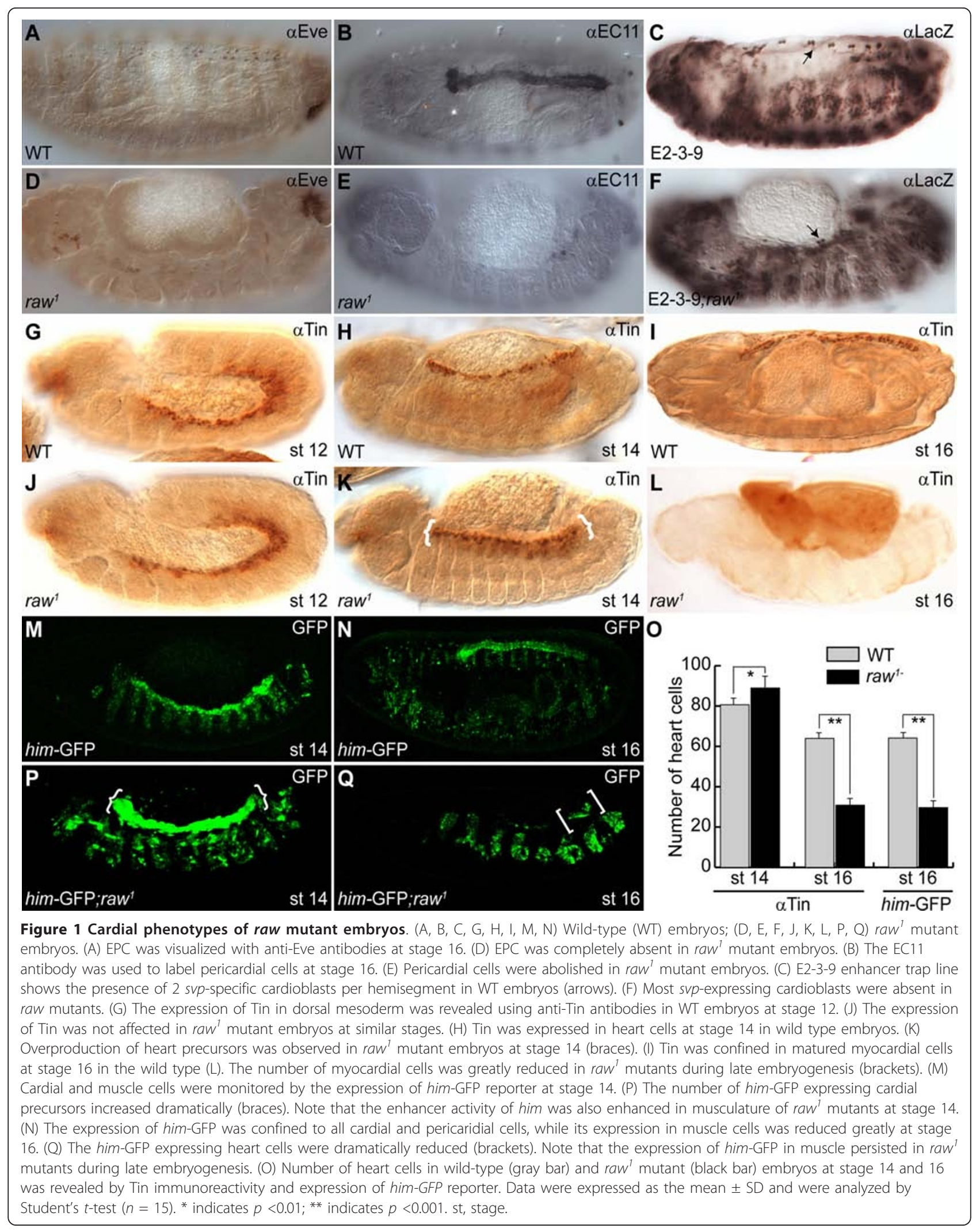




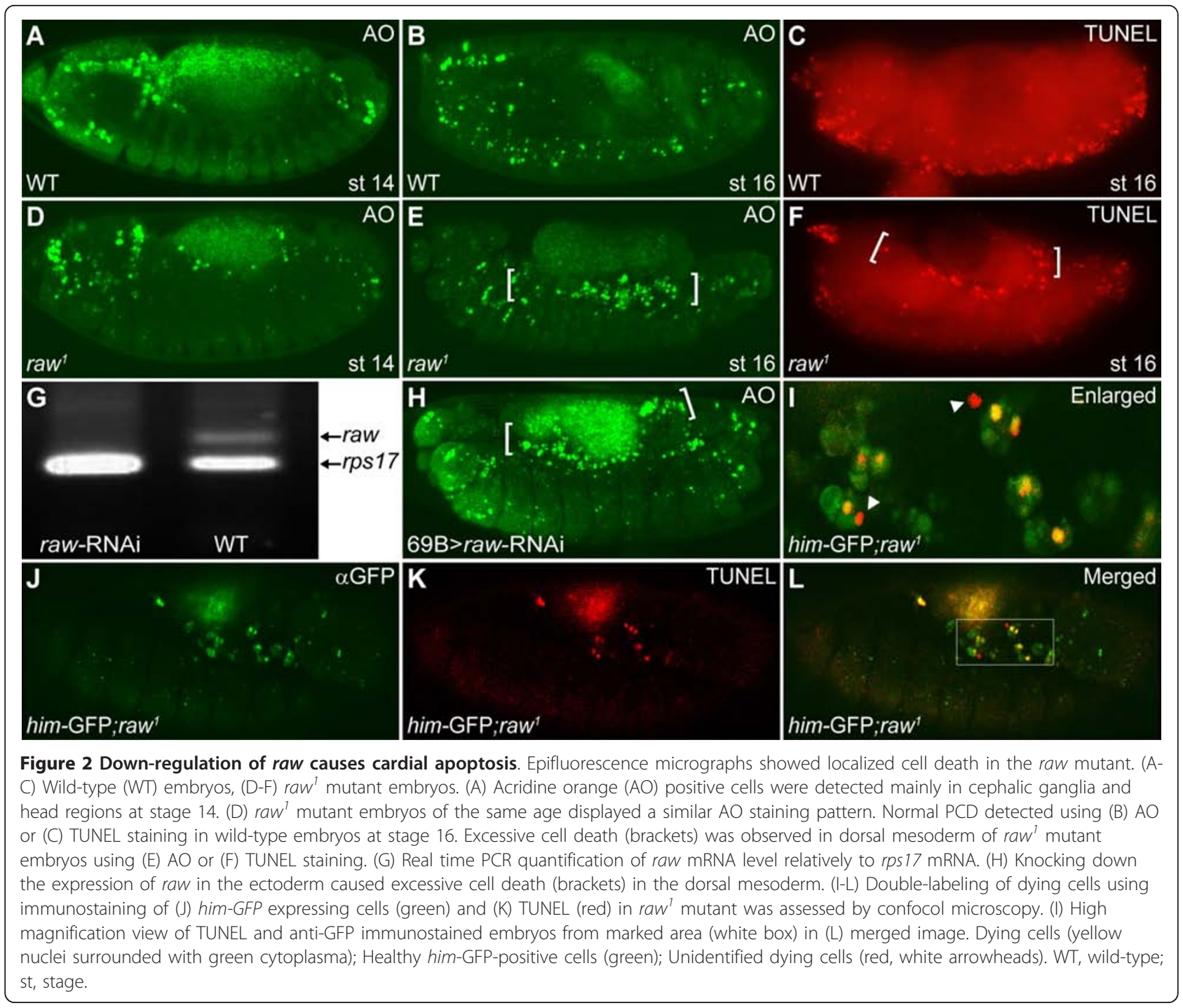

him-GFP-expressing cardial and muscle cells, we noticed few unidentified TUNEL-positive cells (Figure 2I, red, arrowheads). Since heart is the major defective tissue in the mesoderm of raw mutant, we have focused our attention on how loss of raw function leads to cardial apoptosis in Drosophila during late embryogenesis.

\section{Function of raw is required in ectoderm}

Previous study showed that mutation of raw resulted in dorsal open phenotype [35]. Ventral denticle belts are also missing in embryos homozygous for the null $\mathrm{raw}^{1}$ allele (Figure 3B, see also [50]). As demonstrated above, lack of raw function leads to apoptosis of dorsal mesodermal tissues, including heart. These findings suggest that raw is a pleiotropic gene which is required for the normal development of multiple tissues in Drosophila. To determine the spatial requirement of $r a w$, we performed rescue experiments by expressing raw using 69B- or 24B-gal4 driver, which direct the expression of UAS$r a w$ in ectoderm or mesoderm respectively $(69 B>r a w$ or $24 B>r a w)$. Consistent with a previous study, we found that the cuticular phenotypes of raw mutant could be rescued by targeted expression of Raw protein under the control of 69B-gal4 (Figure 3A, C, E, see also [50]). In fact, $55 \%$ of the raw mutant flies survived to adulthood after rescue of the cuticular phenotype (data not shown). By contrast, expression of the UAS-raw transgene driven by $24 B$-gal4 failed to restore the dorsal open or the loss of denticle belt phenotypes (Figure 3D and 3E). Conversely, we were able to replicate the cuticular phenotype by knocking down endogenous raw using a 69B-gal4 driver (Figure 3F). However, no cuticle defect was observed when the raw-RNAi was driven with the pan-mesoderm driver 24B-gal4 (Figure 3G).

Rescue experiments were also conducted to determine whether raw functions cell-autonomously for the survival 

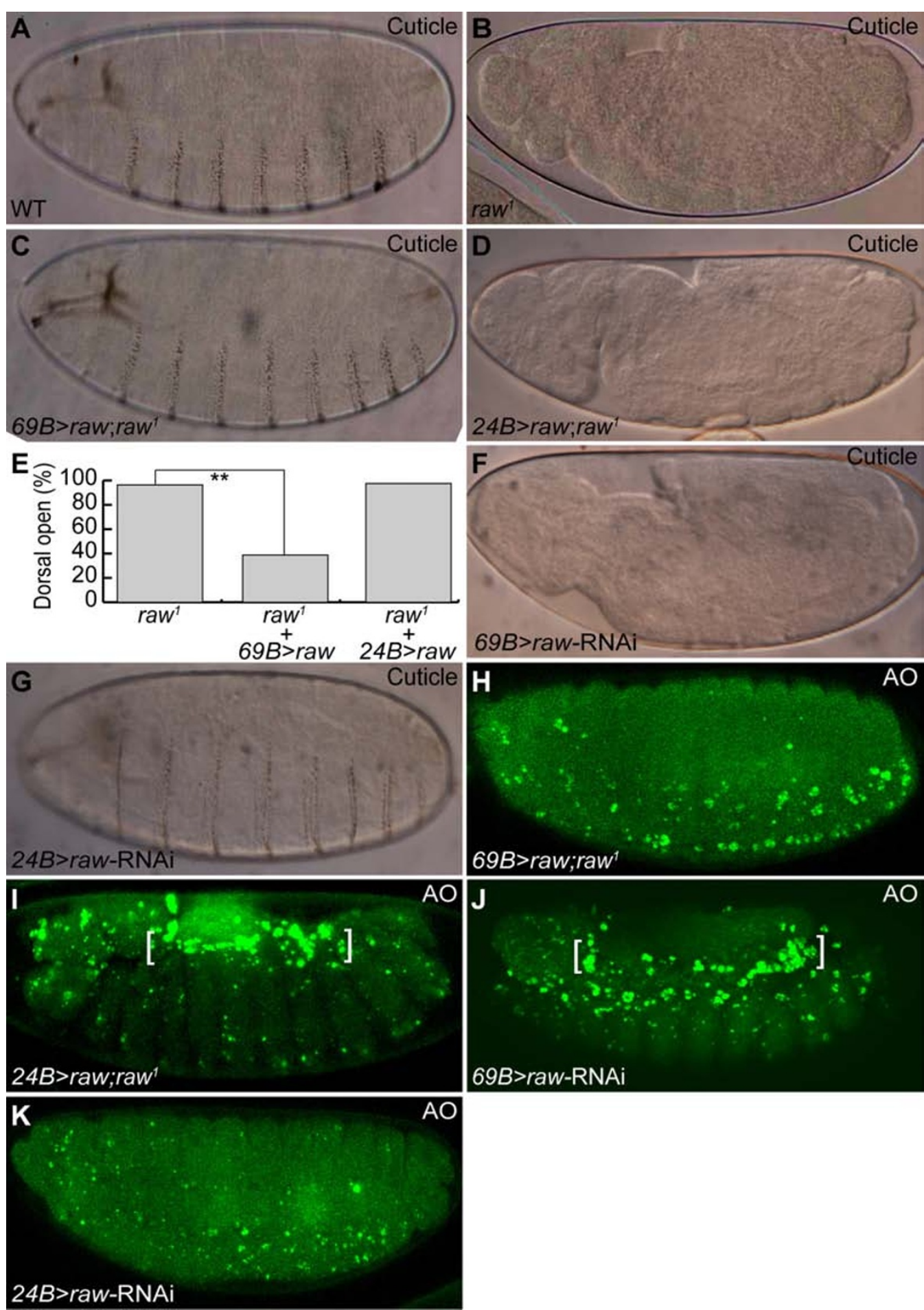

Figure 3 Ectodermal raw is sufficient for the normal development of Drosophila. (A-D, F, G) Cuticular phenotype. (A) Wild type (WT). (B) raw'. (C) UAS-raw; raw /69B-gal4; raw'. (D) UAS-raw; raw'/24B-gal4; raw'. (E) Bar chart showing that ectodermally, but not mesodermally,

expressed raw suppressed dorsal open phenotype in raw mutants. Statistical analysis of the percentage of embryos with dorsal open phenotype

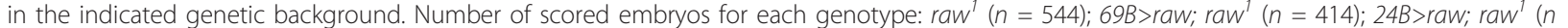
= 324). Chi-square test, ${ }^{* *}$ indicates $p<0.001$. (F) UAS-raw/69B-gal. (G) UAS-raw-RNAi/24B-gal4. (H-K) Apoptosis phenotype revealed by AO staining. Ectodermally $(H)$, but not mesodermally (I), expressed raw blocked cardial apoptosis (brackets) in raw mutants. raw-RNAi transgene driven by 69B-gal4 (J), but not 24B-ga/4 (K), caused cardial apoptosis (brackets). 
of mesodermally-derived tissues. We found that epidermal expression of raw suppressed cardial apoptosis under raw mutant background (Figure $3 \mathrm{H}$ ). However, mesodermallyoverexpressed raw was unable to completely inhibit cardial apoptosis in the raw mutant (Figure 3I, brackets). Additionally, transgenic raw-RNAi construct driven by 69B-gal4, but not 24B-gal4, produced a cardial apoptosis phenotype (Figures $2 \mathrm{H}$, 3J vs. Figure $3 \mathrm{~K}$, brackets). These results strongly suggest that ectodermal Raw is sufficient for the proper development of Drosophila.

\section{Raw affects Dpp and $\mathbf{W g}$ signalings}

Having established that ectodermal Raw functions in a cell non-autonomous manner to affect the viability of dorsal mesodemal cells, we speculated that ectodermallysecreted factors might be responsible for the cardial apoptosis observed in the raw mutant. In this regard, Wg and Dpp are good candidates because they are essential for patterning dorsal mesodermally-derived tissues, including cardial progenitor cells. Previous study has shown that Dpp is ectopically expressed at the dorsal epidermis of raw mutant embryos [35]. Using a $d p p$-LacZ reporter, we confirm that Dpp signaling is ectopically activated in raw mutant embryos at stage 14 (Figure 4A, B vs. Figure 4C, D). To further determine if Dpp signaling is altered in raw mutants, we conducted immunocytochemistry experiments using a monoclonal antibody against pMad [43], a Dpp-activated Smad protein. pMad immunoreactivity was detected as a broad band in the dorsal ectoderm of both wild-type and raw mutant embryos at stage 13 (Figure 4E and 4G). The broad band expression pattern disappeared in wild-type embryos but not in the dorsal ectoderm of raw mutants at stage 14 (Figure 4F vs. 4H, brackets). Moreover, optical sectioning through the stained embryo showed the same broad band expression pattern of pMad in mutant but not in wild-type embryos (Figure $4 \mathrm{I}$ and $4 \mathrm{~K}$, brackets). At late stages, pMad was not detected in the dorsal ectoderm of either mutant or wild-type embryos (Figure 4J and 4L).

Immunostaining was also used to investigate if Wg signaling is affected by the raw mutation. We found that Wg was expressed in transverse striped domains of the ectoderm along the anteroposterior axis in wild type embryos at stages 13 (Additional File 2, Fig. S2). The expression pattern of Wg was not altered in raw mutants by stage 13 . However, the expression level of Wg had significantly decreased in raw mutants at stage 14 (Additional File 2, Fig. S2). At stage 16, residual Wg was detected in the ectoderm of wild-type embryos, but was completely absent in raw mutant embryos (Additional File 2, Fig. S2).

\section{Ectopic Dpp signaling promotes cell death}

To investigate if down-regulation of Wg signaling causes cardial apoptosis, we conducted a temperature shift experiment using a temperature-sensitive $w g^{I L 114}$ allele. Inactivation of $\mathrm{Wg}$ at 9-15 hr, the time window during which Wg expression is missing in raw mutant embryos, did not cause the cardial apoptosis seen in raw mutant embryos, and overexpression of $w g$ did not suppress the apoptosis phenotype under raw mutation background (Additional File 2, Fig. S2). We thus concluded that defective Wg signaling is not the cause of cardial apoptosis.

We then turned our attention to the question of whether ectopic Dpp signaling can induce cardial apoptosis, and found that overexpression of $d p p$, using $69 B$ gal4 $(69 B>d p p)$, phenocopied the raw mutant phenotypes (Figure 5A-F). For instance, him-GFP expressing heart cells were overproduced at stage 14, but they had disappeared in $69 B>d p p$ embryos at stage 16 (Figure $5 \mathrm{~A}-\mathrm{B}$ and Figure 5D-E). Naked cuticle and dorsal open phenotypes were also observed in $69 B>d p p$ embryos (Figure 5C). However, unlike raw mutant embryos in which only him-GFP expressing cardioblasts were lost, both heart and muscle cells were completely abolished in $69 B>d p p$ embryos at stage 16 (Figure $1 \mathrm{Q}$ and $5 \mathrm{E}$ ). This loss of him-GFP-expressing myoblasts and cardioblasts in $69 B>d p p$ embryos is very likely to have resulted from apoptosis, because extensive cell death was detected throughout the entire $69 B>d p p$ embryos, whereas dead cells were located mainly in the dorsal mesoderm in raw mutants (Figure $2 \mathrm{E}$ and $5 \mathrm{~F}$ ). The difference in the apoptosis phenotype between raw mutant and $69 B>d p p$ embryos might reflect the fact that the ectopic $d p p$ was mainly found in the dorsal ectoderm of raw mutant whereas $d p p$ was expressed in the entire ectoderm under the control of 69B-gal4. These findings reinforce our hypothesis that Dpp activity is correlated with cardial apoptosis.

Dpp is a secretory protein which can be transduced to the underlying mesoderm. If the apoptotic phenotype is associated with the ectopic Dpp activity, it would expect that mesodermal overexpression of Dpp should replicate the apoptosis phenotypes seen in $69 B>d p p$ embryos. Indeed, mis-expression of $d p p$ in mesoderm using a pan mesodermal driver, 24B-gal4, induced excessive cell death accompanied with elimination of him-GFP-expressing cells during late developmental stages (Additional File 3, Fig. S3). These results suggest that excessive $d p p$ in ectoderm or mesoderm can cause apoptosis and removal of cardial cells.

Dpp transduces its signaling by binding to a heteromeric type I/type II transmembrane serine/threonine kinase receptor complex, encoded by $t k v$ and punt. To further demonstrate that ectopic Dpp signaling is the cause of apoptosis in raw mutants, we targeted overexpression of a constitutively-active form of $t k v, t k v$, using a $24 B$-gal4 driver $\left(24 B>t k v^{C A}\right)$. We found that 


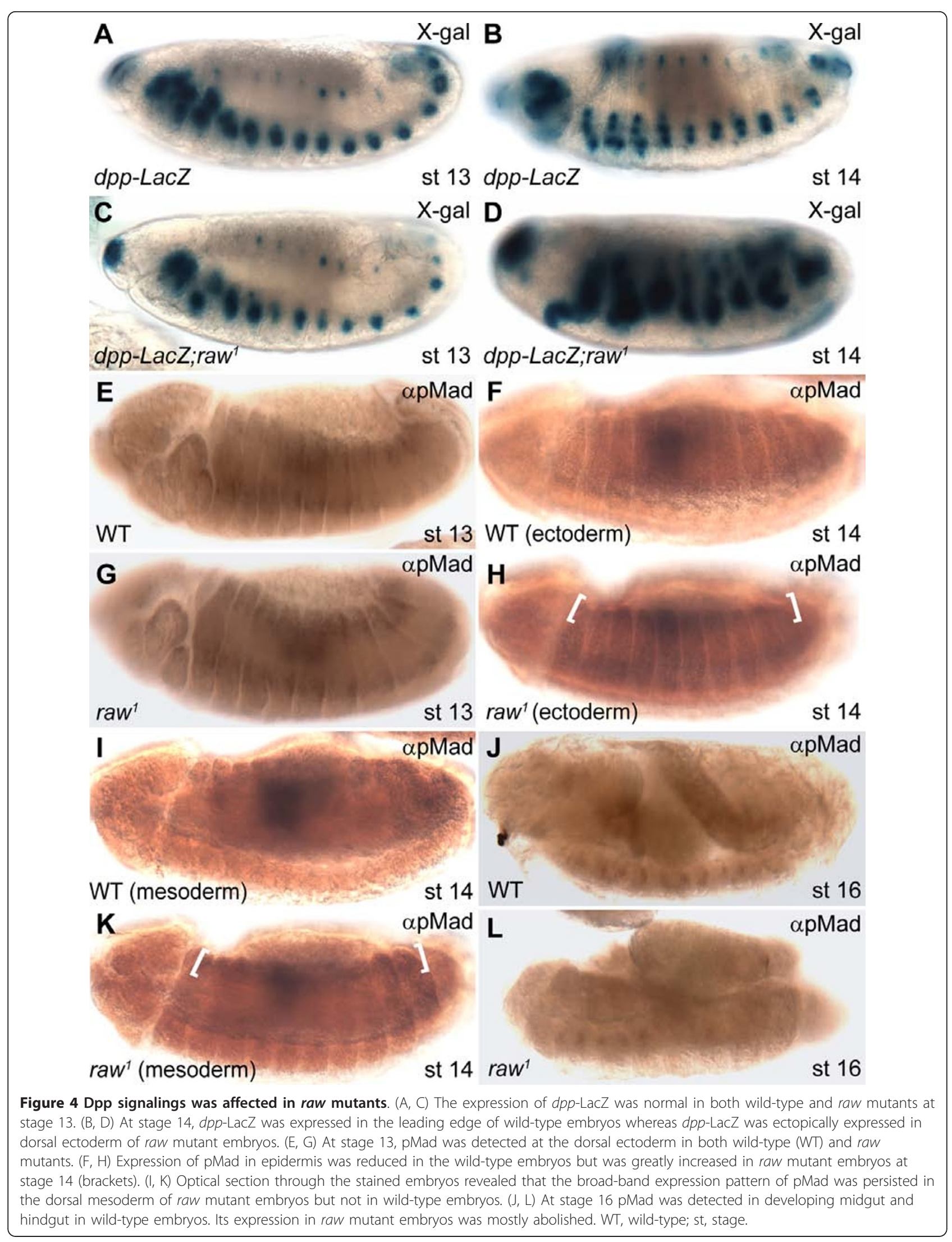




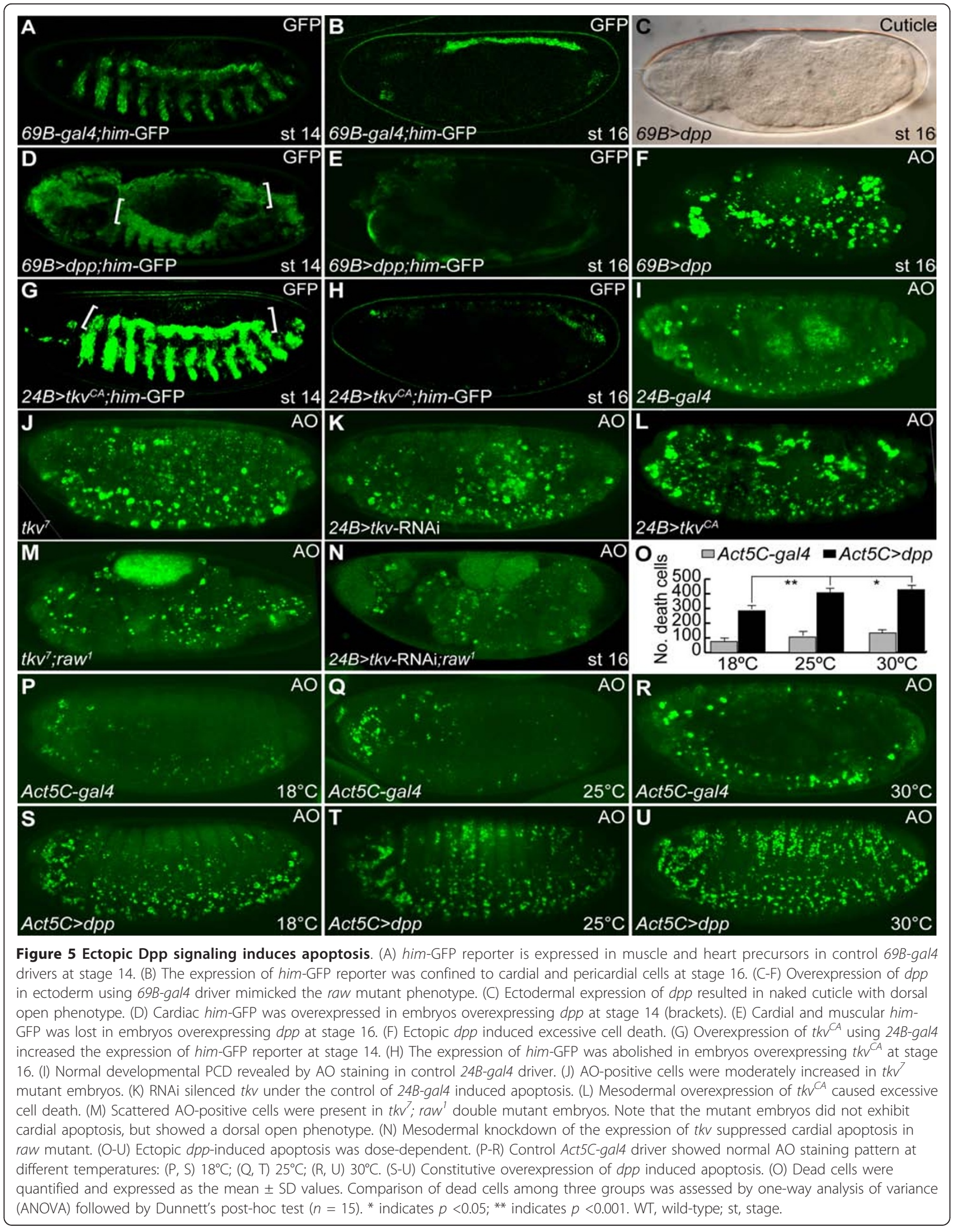


$24 B>t k v^{C A}$ embryos exhibited the same phenotype as $69 B>d p p$ or $24 B>d p p$ embryos, in which him-GFPexpressing cardial cells were increased in $24 B>t k v^{C A}$ embryos at stage 14 (Figure 5G), while him-GFP-expressing heart and muscle cells were eliminated at stage 16 (Figure $5 \mathrm{H}$ ). Extensive cell death was also detected throughout $24 B>t k v^{C A}$ embryos (Figure $5 \mathrm{~L}$ ).

In a complementary experiment, we depleted the expression of $t k v$ by RNAi using a transgenic fly carrying inverted repeats corresponding to $t k v$, under the control of a UAS sequence inducible by $24 \mathrm{~B}$-gal4 $(24 B>t k v-R N A i$, Additional file 4, Fig. S4). Down-regulation of mesodermal $t k v$ induced a moderate degree of apoptosis in which the AO-positive cells were randomly distributed in the entire $24 B>t k v$-RNAi embryos (Figure $5 \mathrm{I}$ vs. $5 \mathrm{~K})$. A similar pattern of scattered cell death was also detected in loss-of-function of $t k v$ mutant embryos (Figure 5J). These data suggest that Dpp is required for cell survival during embryogenesis in Drosophila. To test whether inhibiting Tkv activity can block excessive Dpp-induced apoptosis, we compared the AO staining pattern in raw, tkv and raw;tkv double mutant embryos. Both $t k v$ and raw;tkv double mutant embryos exhibited the same scattered AO staining pattern, unlike the cardial apoptosis phenotype observed in raw mutant (Figure $2 \mathrm{E}$ vs. 5J and $5 \mathrm{M})$. Moreover, mesodermal knockdown of the expression of $t k v$ was able to suppress cardial apoptosis under the raw mutant background (Figure $5 \mathrm{~N}$ ). These results indicate that ectopic Dpp induced apoptosis is tranduced through Tkv.

As above-mentioned, ectopic $t k v$ expression promoted apoptosis in $24 B>t k v^{C A}$ embryos, indicating that Dpp can function as a pro-apoptotic signaling. To directly test this hypothesis, Dpp was constitutively overexpressed under the control of the ubiquitous Act5C-gal4 driver $(A c t 5 C>d p p)$. In control Act5C-gal4 embryos, $\mathrm{AO}$ staining revealed that apoptotic cells were mainly present in the ventral nerve cord and head regions at stage 16 (Figure 5P-R). Raising the temperature slightly increased the amount of cell death in the central nervous system of late Act5C-gal4 embryos (Figure 5O-R). Constitutive overexpression of Dpp caused a remarkable degree of apoptosis in Act5C $>d p p$ embryos (Figure 5S$\mathrm{U})$. Notably, increasing the $d p p$ expression level by raising the temperature significantly increased the number of dying cells (Figure 5O-U). This result suggests that Dpp can function as a pro-apoptotic signaling in a dosedependent manner.

\section{Dpp induced apoptosis is mediated through DTAK1}

As shown above, reducing Tkv activity successfully blocked $d p p$-mediated cardial apoptosis in raw mutants (Figure $5 \mathrm{M}$ and $5 \mathrm{~N}$ ). This data encouraged us to further examine whether overexpression of brinker (brk), a transcriptional repressor of Dpp, could inhibit the cardial apoptosis phenotype in raw mutants. Overexpression of brk using $24 B$-gal4 driver $(24 B>b r k)$ caused a moderate degree of apoptosis (Figure 6A). The AO staining pattern in $24 B>b r k$ embryos was similar to the pattern in $t k v^{7}$ and $24 B>t k v-$ RNAi embryos (Figures 6A, $5 \mathrm{~J}$, and $5 \mathrm{~K}$ ), suggesting that ectopic brk-induced apoptosis was likely to be a result of the inhibition of Dpp signaling. Unexpectedly, mis-expression of brk in mesoderm failed to suppress the cardial apoptosis phenotype of the raw mutant (Figure 6B, brackets), suggesting that the suppressor activity of Brk may not be strong enough to block Dpp-mediated cardial apoptosis. Nevertheless, it is equally possible that Dpp-induced apoptosis is mediated through a distinct pathway which can not be repressed by Brk.

A previous study showed that TGF- $\beta$ activated kinase 1 (TAK1), a member of the JNKK kinase superfamily that activates the JNK cascade, can transduce TGF- $\beta$ signaling and induce apoptosis in vertebrates [51]. To determine if TAK1 induces apoptosis in response to Dpp signaling, a Drosophila TAK1 homolog, DTAK1, was mis-expressed using either Act5C-or $24 B$-gal4. Ectopic DTAK1 activity was sufficient to induce apoptosis in embryos (Figure 6C and 6D). In contrast, ectodermal or mesodermal overexpression of a dominant negative form of $d T A K 1\left(d T A k 1^{D N}\right)$ was capable of suppressing developmental programmed cell death (Figure $6 \mathrm{E}$ and $6 \mathrm{~F}$ ). Constitutive overexpression of $d T A K 1^{D N}$ also effectively inhibited ectopic Dpp-induced apoptosis (Figure 6G). Moreover, mesodermally overexpressed $d T A K 1^{D N}$ suppressed cardial apoptosis under the raw mutation background (Figure 6H). Taken together, our results clearly demonstrate that DTAK1 is a downstream effector of Dpp-mediated cardial apoptosis.

\section{DTAK1 induces JNK dependent apoptosis}

Although our data suggest that $d T A K 1$ acts downstream of $d p p$ to promote apoptosis, it is interesting to note that $d T A K 1$ stimulates $d p p$ expression, suggesting that $d p p$ is a downstream target of $d T A K 1$ [29]. Despite the discrepancy in these epistatic relationships, these observations strongly suggest that $d T A K 1$ and $d p p$ act in the same genetic pathway. Using a $d p p$-LacZ reporter, we found that $d T A K 1$ activated the transcription of $d p p$ to the same degree as basket (bsk), the Drosophila Jun amino-terminal kinase (DJNK) homolog encoded gene which activates the expression of $d p p$ in the dorsalmost epidermal cells (Figure 7A-C). These results indicate that $d T A K 1$ acts upstream of $d p p$. Since $d p p$ is also activated by $b s k$, these results suggest that $d T A K 1$ activates $b s k$ and thereby $d p p$. Moreover, we found that overexpression of $d T A K 1$ using $24 B$-gal4 driver stimulated the expression of pMad in the entire mesoderm 

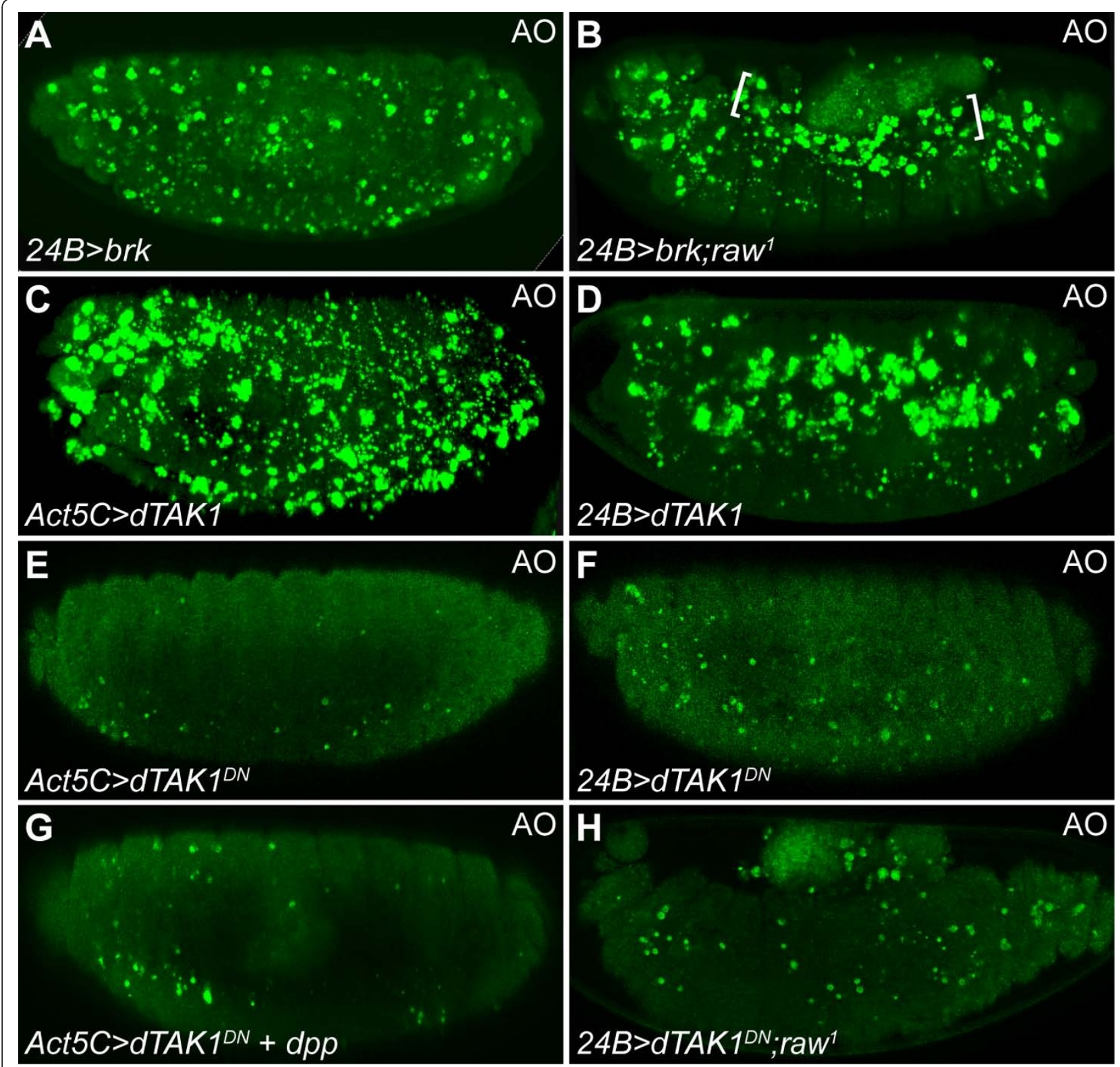

Figure 6 Ectopic Dpp-induced apoptosis is mediated through DTAK1. (A) Ectopic expression of brk using 24B-ga/4 induced cell death. (B) Mesodermal overexpression of brk did not suppress cardial apoptosis in raw mutants (brackets). (C, D) Excessive cell death was induced by overexpression of dTAK1 using Act5C- or 24B-gal4 drivers. (E, F) Overexpression of dominant negative $d T A K 1^{D N}$ using Act5C- or 24B-gal4 drivers inhibited apoptosis. (G) Constitutively-expressed $d T A K 1^{D N}$ suppressed ectopic dpp-induced apoptosis. (H) Mesodermal expression of $d T A K 1^{D N}$ inhibited cardial apoptosis in raw mutants.

(Figure 7D vs. Figure 7E), indicating that the ectopic Dpp induced by DTAK1 can transduce its own signaling through Tkv and result in the expression of ectopic pMad. As Dpp can transduce its signaling through DTAK1 to promote apoptosis (Figure 6G), the ectopic Dpp induced by DTAK1 is likely to autoregulate itself through DJNK. In other words, a positive autoregulatory loop for Dpp expression is formed when DTAK1 is activated.
The JNK cascade is a well-known pro-apoptotic signaling that participates in stress-related apoptosis in Drosophila. Together with the fact that TAK1 can activate the JNK cascade, the autocrine Dpp is expected to enhance the activity of DTAK1 and thereby hyperactivitates the DJNK pathway. In other words, the ectopic $d p p$-induced cell death could be a consequence of hyperactivated JNK signaling. To test this hypothesis, bsk was overexpressed in the entire embryo or 

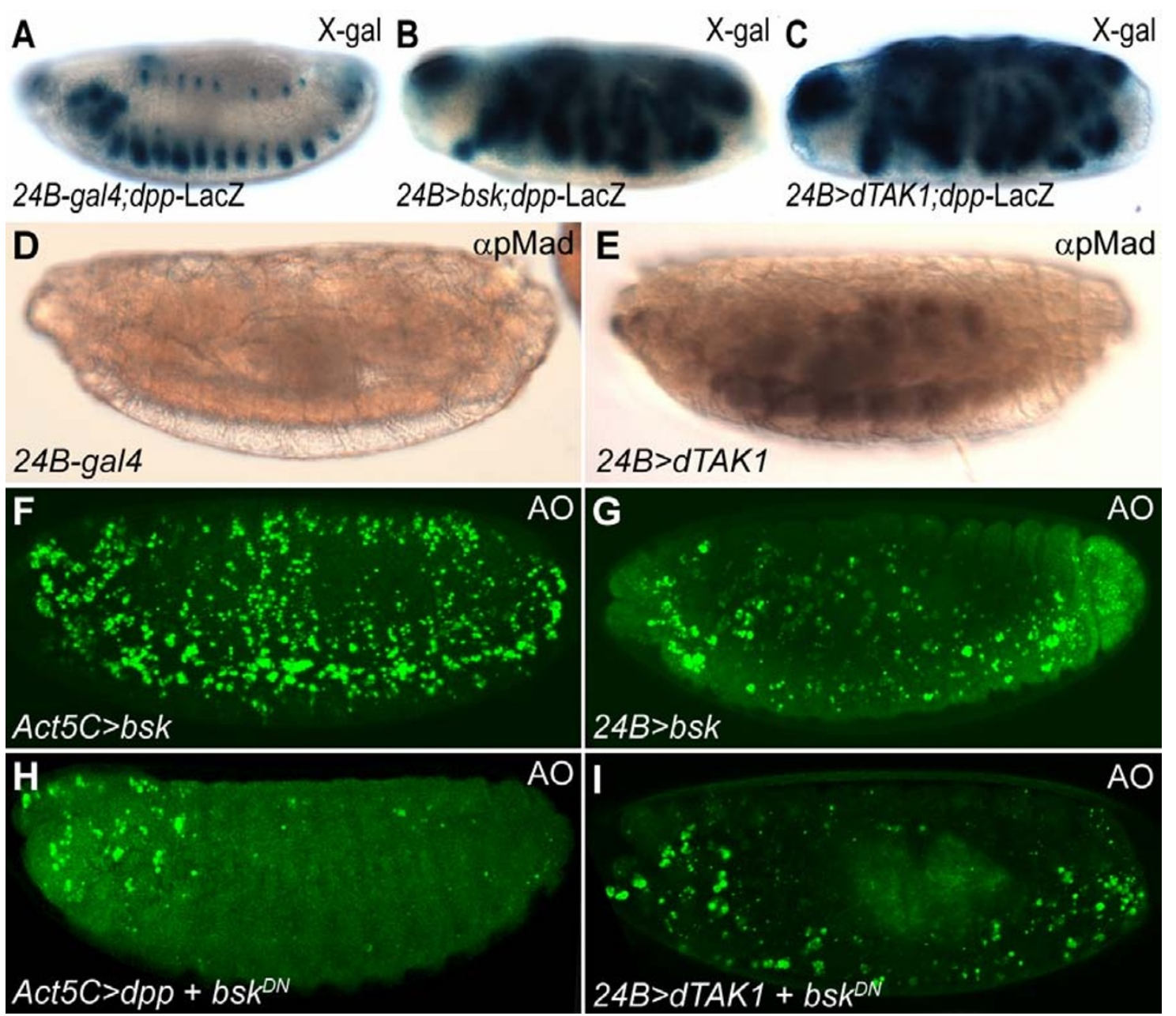

Figure 7 DTAK1 activates DJNK pathway. (A) dpp-LacZ showed a typical expression pattern in dorsal and lateral spots along the anteriorposterior axis in control 24B-gal4 driver embryos. (B) Mesodermal bsk induced the expression of dpp-LacZ. (C) dpp-LacZ reporter was ectopically expressed in 24B>dTAK1 embryos. (D) pMad was not ectopically expressed in control 24B-gal4 driver. (E) Targeted overexpression of dTAK1 in mesoderm induced ectopic pMad. ( $F, G)$ Excessive apoptosis was induced by the expression of bsk using Act5C-or 24B-gal4 drivers. (H) Expression of $b s k^{D N}$ inhibited ectopic dpp-induced apoptosis. (I) Mesodermal expression of $b s k^{D N}$ inhibited dTAK1-induced cell death.

specifically in mesoderm using either $A c t 5 C$ - or $24 B$ gal4 driver (Act5C >bsk; 24B>bsk). Ectopic bsk activity dramatically increased levels of apoptosis in the embryos (Figure 7F and 7G). Conversely, overexpression of a dominant negative $b s k, b s k^{D N}$, again using either $A c t 5 C$ or 24B-gal4 driver, was able to suppress ectopic Dpp/ DTAK1-induced apoptosis (Figure 7H and 7I). These results demonstrate that Dpp exerts its pro-apoptotic functions through DTAK1 as well as through DJNK.

\section{Overexpression of anti-apoptotic P35 or dominant} negative P53 blocks Dpp induced apoptosis

As demonstrated above the missing of cardial cells in raw mutants is likely to be a consequence of excessive $d p p$ induced apoptosis. If this is the case, we expect that blocking cell death would reverse the cardial apoptosis phenotype in raw mutant embryos. The baculovirus P35 protein has been shown to suppress normal and induced apoptosis by inhibiting caspases in animals [36]. We found that overexpression of the anti-apoptotic P35 could prevent apoptotic cell death induced by either ectopic Dpp or DTAK1 (Figure 8A and 8C). P35 also significantly reduced the death of heart cells under the raw mutation background (Figure 8E). Moreover, it has been shown that aberrant JNK-induced apoptosis is mediated through P53 (reviewed in [52]). To further demonstrate if Dpp/DTAK1-induced apoptosis is P53dependent, a dominant negative form of $p 53\left(p 53^{D N}\right)$ was ectopically expressed using either Act5C- or $24 B$ gal4 driver $\left(A c t 5 C>p 53^{D N}\right.$ or $\left.24 B>p 53^{D N}\right)$. We found that overexpresion of $p 53^{D N}$ suppressed Dpp- or DTAK1-induced apoptosis successfully (Figure 8B and 

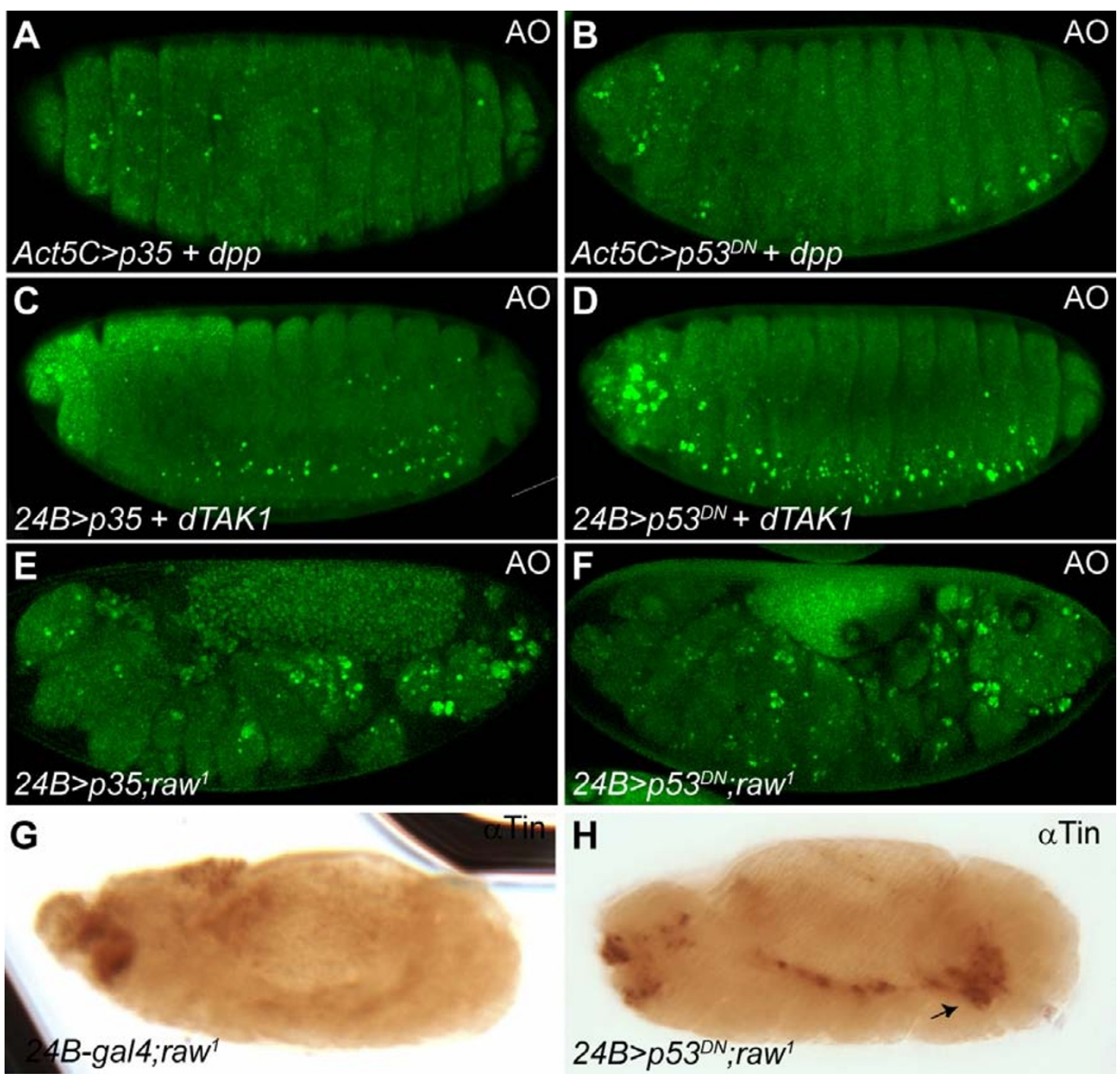

Figure 8 JNK induced cardiac apoptosis is p53 dependent. (A, B) Constitutive overexpression of $p 35$ or dominant negative p53 $3^{D N}$, using Act5C-gal4 driver, antagonized dpp-induced apoptosis. (C, D) Mesodermal expression of $p 35$ or p53 ${ }^{D N}$ suppressed dTAK1-induced apoptosis. (E, F) Overexpressed $p 35$ or $p 53^{D N}$ driven by $24 B$-gal4 suppressed apoptosis in raw mutants. (G) Loss of raw function resulted in loss of heart cells as revealed by anti-Tin antibodies in control 24B-gal4 driver. $(H)$ Excessive Tin-positive heart cells were present when $p 53^{D N}$ was expressed in raw mutants (arrow).

8D), suggesting that Dpp/DTAK1-induced apoptosis is mediated through P53. We also showed that mesodermal overexpression of $\mathrm{P} 53^{\mathrm{DN}}$ significantly reduced the death of heart cells in raw mutant embryos (Figure 8F). Consistent with the above observation, immunostaining experiments revealed that mesodermally-overexpressed P53 ${ }^{\mathrm{DN}}$ was able to rescue Tin-positive cardial cells in raw mutant embryos (Figure $8 \mathrm{G}$ and $8 \mathrm{H}$ ). In fact, superfluous Tin-positive cells were usually observed when cardial apoptosis was blocked by ectopic P $53^{\mathrm{DN}}$ expression (Figure 1I vs. Figure $8 \mathrm{H}$, arrow), suggesting that the overspecified cardial cells during mid-embryogenesis in raw mutants survived when apoptosis was blocked.
These results also indicate that the Dpp-DTAK1-DJNK mediated apoptosis pathway is likely to be P53dependent.

\section{Discussion}

Function of Raw in DJNK signaling pathway

Analysis of the amino acid sequence of Raw revealed that it does not comprise any specific functional domain or motifs. Despite the fact that the C-terminus of Raw protein is rich in glutamine residues, a characteristic feature of some transcription factors, Raw protein was mainly detected in cytoplasma, suggesting that it may not play a role in transcriptional regulation. The most 
prominent feature of Raw protein is that it contains two duplicated domains with a 32 amino acid core repeat (designated as Raw repeats) that is highly conserved in invertebrates [35]. The structure of Raw is currently unavailable, so no further information regarding the function of Raw can be deduced through sequence analysis.

Epistasis analysis has demonstrated that raw negatively regulates jra activity in parallel to DJNK signaling in the epidermis of Drosophila [35,50]. As demonstrated above, Dpp-induced apoptosis is mediated through activation of DJNK signaling (Figure $7 \mathrm{H}$ ). If raw can negatively regulate DJNK, it would be expected that forced expression of raw would inhibit the DJNK cascade and reverse the apoptotic phenotype in the raw mutant. Ectodermal overexpression of raw did rescue the cardial apoptosis in raw mutants (Figure $3 \mathrm{H}$ ). Nevertheless, targeted expression of raw in the mesoderm was unable to prevent cell death under the raw mutation background (Figure 3I). Why is the effect of mesodermal Raw so different from that of ectodermal Raw? As demonstrated above, Dpp can trigger its own expression in the mesoderm through DTAK1 and DJNK (Figure 7A-E). This auto-regulated Dpp is expected to further enhance DJNK activity, which may out-compete the suppressive activity of Raw. This might explain why mesodermal expression of raw was unable to suppress raw mutationinduced apoptosis. However, if the initial Dpp signaling in ectoderm is suppressed then it will not initiate the autocrinal Dpp signaling in mesoderm and result in cell death. Consistent with our notion, we found that ectodermally-overexpressed raw suppressed the expression of pMad in raw mutants. In contrast, targeted expression of raw in mesoderm did not inhibit the expression of pMad under the raw mutation background (Additional File 5, Fig. S5).

\section{Dpp functions as survival and pro-apoptotic signals}

The morphogenetic function of Dpp patterns cell fates across the developing field by forming a gradient which provides position identity for the receiving cells. Similarly, it seems that Dpp controls the viability of cells in the same concentration-dependent manner. Mutant cells deprived of Dpp signaling are lost from wing disc epithelium due to DJNK activation and apoptosis $[30,53]$. These observations have suggested that Dpp functions as a survival factor by preventing activation of the DJNK-dependent apoptotic pathway. Additionally, down-regulation of mad activated JNK and caspase-3, indicating that Dpp functions as a survival factor mediated through Mad [54]. Consistent with these findings, we found that down-regulation of $t k v$ or overexpression of $b r k$ induced moderate cell death in embryos (Figure 5J, K and 6A). Nevertheless, Dpp seems to act as a double-edged sword because increased Dpp signaling induces DJNK-mediated apoptosis in the proximal wing [30]. Similarly, in this study, we show that ectopic $d p p$ or $t k v^{C A}$ expression promotes DJNK-mediated apoptosis in embryos (Figure 5F and 5L). Moreover, the apoptotic propensity of Dpp is proportional to its own expression level (Figure 5O and 5S-U). Taken together, these results suggest that Dpp can act as both survival and death signals and thus, an appropriate expression level of Dpp is indispensible for the survival of cells during development.

\section{TAK1 is a key transducer that mediates ectopic Dpp induced apoptosis}

TAK1 is also a member of the MAPKKK family, which was originally identified as a mediator of TGF- $\beta$ signaling pathway [55]. It has also shown to regulate a great variety of cellular processes through activating many down-stream kinase cascades, including I-kappa B kinase complex (IKK), p38 MAPK, JNK, and AMP-activated protein kinase (AMPK) (reviewed in [56]). Unlike the canonical pathway in which members of TGF- $\beta$ family elicit phosphorylation of Smad proteins, activation of TAK1 was found to function in a receptor kinase-independent manner [26]. The existence of the non-canonical pathway may explain why mesodermal overexpression of brk was unable to block cardial apoptosis in raw mutants (Figure 6B), because it seems that brk only suppresses Dpp target genes containing Mad consensus binding sites [57]. Interestingly, mesodermal pMad was increased in raw mutant or in embryos overexpressing $d T A K 1$ (Figure 4K, and 7E), indicating that both canonical and non-canonical pathways were activated simultaneously in response to ectopic Dpp signaling. The fact that apoptotic cell numbers increased dramatically in raw mutants or in embryos overexpressing dTAK1 (Figure 2E, F, 6C and 6D) suggests that the non-canonical pathway suppresses the canonical pathway when cells are exposed to excessive Dpp levels. Together with the fact that the apoptotic propensity of Dpp is dose-dependent (Figure 5O), these data imply that higher levels of Dpp elicit stronger DTAK1 activity and result in apoptosis. In support of this argument, homozygous raw mutant animals had significant apoptosis in the dorsal-most tissues, such as the heart, where Dpp activity is at its peak (Figure 2E and 2F), and global overexpression of Dpp signaling led to ubiquitous cell death (Figure 5F, L and 5S-U).

\section{Late Dpp signaling in heart development}

Compared to the early function of Dpp in patterning cardiogenic mesoderm, the function of Dpp during late cardiogenesis is less explored. Previously, studies had showed that numbers of various pericardial cell types, 
but not cardial cells, were increased in fly embryos with the $d p p^{d 6}$ mutant allele, whose expression was not maintained in the dorsal ectoderm during germ-band retraction $[23,24]$. The expression of the mitosis marker phospho-histone 3 was concomitantly increased in the $d p p^{d 6}$ mutant, suggesting that Dpp restricts the proliferation of pericardial cells specifically during late cardiogenesis [24]. As shown above, $d p p$ signaling is ectopically-activated at stages 14-15 in raw mutants (Figure 4D, E and 4K, see also [58]); this also gives us a good opportunity to decipher late Dpp function in the developing heart of Drosophila. As demonstrated above, ectopic Dpp signaling leads to both overproliferation of cardial cells during mid-embryogenesis and cardial apoptosis during late embryogenesis because overexpression of $d p p$ or $t k v^{C A}$ replicated the raw mutant phenotypes (Figure 5A-H). Interestingly, both cardial and pericardial cells were eliminated in either raw mutant, $69 B>d p p$ or $24 B>t k v^{C A}$ embryos (Figure $1 \mathrm{D}-\mathrm{F}, \mathrm{L}, \mathrm{O}, \mathrm{Q}$, $5 \mathrm{E}$ and Figure $5 \mathrm{H}$ ). Our data contradict the observation that targeted overexpression of $t k v^{C A}$ under the control of cardioblast-specific tinC $\Delta 4$-gal4 did not reduce the number of cardial cells significantly [24]. The disparate results may be due to differences in the temporospatial expression of the transgene driven by different gal4 drivers. Alternately, if the pro-apoptotic propensity of Dpp is concentration-dependent (Figure $5 \mathrm{O}$ and $5 \mathrm{~S}-\mathrm{U}$ ), as discussed above, it is also possible that the expression of $t k v^{C A}$ driven by tinC $\Delta 4$-gal4 is not strong enough to trigger the apoptosis response in cardioblasts.

\section{Model for raw mutation mediated apoptosis}

Based on our data, we propose a model to depict the genetic pathways involved in raw mutation-mediated apoptosis (Figure 9). At stage 14, Dpp activity in leading edge cells activates cardiogenic factors in the underlying mesoderm which are essential for the differentiation of dorsal mesodermally derived tissues, including the heart. Deficits in Raw function cause overexpression of $d p p$ which increases the activities of cardiogenic factors and results in overgrowth of cardial cells at stage 14 . At stage 15, the expression of Dpp in the LE cells is maintained by the DJNK cascade, and heart cells are continuously differentiated by the function of cardiogenic factors in wild-type embryos. In raw mutant embryos, ectopic Dpp activates DTAK1 which triggers the expression of Bsk as well as Dpp. The induced Dpp functions in an autocrine manner to further enhance activity of Bsk and eventually lead to P53-mediated apoptosis.

In support of our model, it has been found that TGF$\beta /$ SMAD signaling exerts its apoptotic function in an autocrine loop manner in rat cardiomyocytes [59]. Induction of cardiomyocyte apoptosis by caspase overexpression has been shown to cause lethality and dilated cardiomyopathy in mice [60]. In contrast, inhibition of cardiomyocyte apoptosis by treating with a caspase

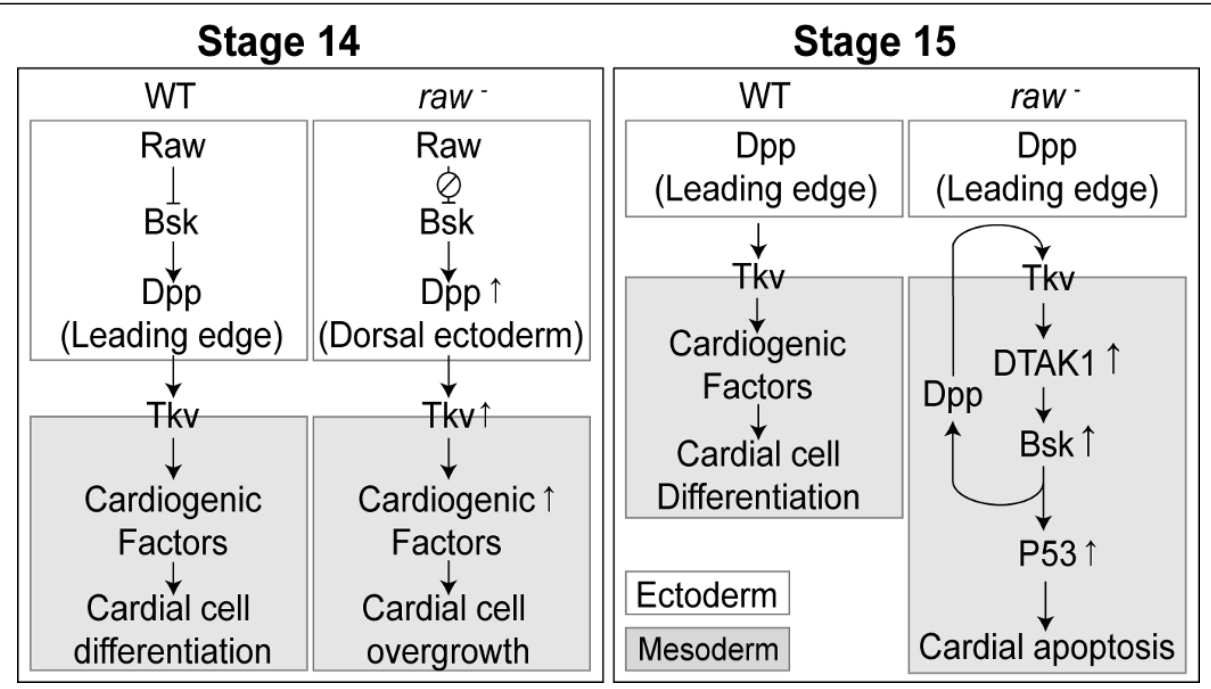

Figure 9 The genetic network that leads to cardiac apoptosis in raw mutation. At stage 14, Raw restricts bsk which limits the expression of $d p p$ at ectodermal leading edge cells. Minimal Dpp activity maintains the activation of cardiogenic factors which are essential for the differentiation of cardial cells. In raw mutation, Bsk is de-regulated which causes overexpression of dpp in dorsal ectoderm at stage 14. Ectopic $d p p$ signaling increases the expression of cardiogenic factors which results in overgrowth of cardial cell types at stage 14 . At stage 15 , while the expression of Dpp in the LE cells gradually decreases, heart cells are continuously differentiated with the function of cardiogenic factors. At stage 15, DTAK1 is activated by the ectopic Dpp signaling in raw mutant. DTAK1 activates DJNK and thereby Dpp. Autocrine Dpp further enhances DJNK and eventually leads to P53-dependent apoptosis. 
inhibitor reduced apoptosis, improved cardiac function, and delayed progression of heart failure in a cardiomyopathy animal model [61]. Prolong haemodynamic overload can up-regulate TGF- $\beta[62,63]$, and death of cardiomyocytes is observed in virtually every myocardial disease (reviewed in [64]. The pathway unraveled in this study is the first report that links ectopic Dpp and DJNK-dependent cardial apoptosis through the noncanonical pathway and dTAK1 activation. Our findings may thus suggest possible medical interventions for human cardiomyopathy.

\section{Conclusion}

By analyzing the heart defect phenotype of raw mutant embryos, we demonstrate that overexpression of $d p p$ lead to cardial apoptosis during late embryogenesis of Drosophila. We also demonstrate that Dpp induces its own expression through dTAK1. The activation of dTAK1 causes the hyperactivation of Drosophila JNK (DJNK) thereby cardial apoptosis. This is the first report that links ectopic Dpp and DJNK-dependent cardial apoptosis through the non-canonical Dpp signaling pathway and dTAK1 activation. Since haemodynamic overload usually up-regulates TGF- $\beta$, a mammalian homolog of Dpp, and death of cardiomyocytes, the pathway delineated in this study may suggest possible medical interventions for human cardiomyopathy.

\section{Additional material}

Additional file 1: Fig. S1. Odd-skipped pericardial cells (OPCs) are missing in raw mutant embryos at late stages. (A) wild-type embryos show the presence of OPCS. (B) OPCs are completely absent in raw mutants.

Additional file 2: Fig. S2. Loss-of Wg function does not lead to localized apoptosis. (A, D) Wg was expressed in a series of ectodermal cells at dorsal and ventral sites of embryos at stage 13. The expression pattern was not altered in raw mutant. (B, E). Lateral expression of Wg became a transverse stripe in the dorsal ectoderm of wild-type embryos. However, its expression decreased significantly in raw mutant embryos at stage 14. (C, F) At stage 16, residual Wg staining was detected in the dorsal epidermis of wild-type embryos, but its expression was completely lost in raw mutants at stage 16. (G) raw mutation shows cadial apoptosis phenotype (brackets). (H) $\mathrm{wg}^{1 / 114}$ is a temperature-sensitive allele that mimics the null wg allele at non-permissive temperatures. Removal of wg function does not lead to cardial apoptosis phenotype in temperature shift experiment using $w^{1 / 1114}$ allele. Incubation times (9-15 hr) were normalized to development at $25^{\circ} \mathrm{C}$. (I) Ectopic wg expression driven by 69B-ga/4, did not suppress cardial apoptosis in raw mutants (brackets).

Additional file 3: Fig. S3. Mesodermally overexpression of Dpp induces raw-like phenotypes. (A, him-GFP reporter was expressed in muscle and heart precursors in 24B-gal4 control flies at stage 14. (B) Expression of him-GFP was limited in heart cells in control 24B-gal4 driver at stage 16. (C) Mesodermal overexpression of dpp induced ectopic heart cells at stage 14. (D) him-GFP expressing heart cells were lost in embryos expressing dpp using 24B-gal4 at stage 16. (E, F) Normal AO staining pattern was observed in 24B-gal4 control driver at stage 14 and 16. (G) Mesodermal overexpression of dpp does not induce apoptosis at stage 14. $(H)$ Excessive cell death was detected in embryos overexpressing dpp using 24B-gal4 at stage 16 .
Additional file 4: Fig. S4. The expression of the endogenous tkv was specifically silenced as compared to $r p s 17$ mRNA in the embryos. RT-PCR products were resolved in 1.5\% agarose gel and visualized with EtBr. WT, wild-type embryos.

Additional file 5: Fig. S5. Ectodermally, but not mesodermlly overexpression of raw suppresses the ectopic pMad in raw mutant. (A) pMad was detected as a broad dorsal band in raw mutant (brackets). (B) Targeted expression of raw using 69B-gal4 inhibited ectopic pMad in raw mutant. (C) Forced expression of raw using 24B-ga/4 can not inhibit ectopic pMad in raw mutant (brackets).

\section{Acknowledgements}

We are grateful to A. Letsou, J. Posakony, L. Marsh, T. Adachi-Yamada, M. Frasch, H. Sun, the Bloomington Stock Center, the Vienna Drosophila RNAi Center, the Drosophila Genomics Resource Center, Fly core of Taiwan and the Developmental Studies Hybridoma Bank for CDNA, fly stocks, antibodies and reagents. This work was supported by grants from the National Health Research Institute (NHRI-EX91-9109SC) and the National Science Council (972311-B-003-003) of the Republic of China.

\section{Authors' contributions}

SAY conducted all the experiments. MTS designed the experiments and wrote the manuscript. All authors read and approved the final manuscript

\section{Competing interests}

The authors declare that they have no competing interests.

Received: 21 June 2011 Accepted: 24 November 2011

Published: 24 November 2011

\section{References}

1. Bier E, Bodmer R: Drosophila, an emerging model for cardiac disease. Gene 2004, 342:1-11.

2. Ocorr K, Perrin L, Lim HY, Qian L, Wu X, Bodmer R: Genetic control of heart function and aging in Drosophila. Trends Cardiovasc Med 2007, 17:177-182.

3. Qian L, Liu J, Bodmer R: Heart development in Drosophila. Bodmer, R. (ed.), Advances in Developmental Biology. Elsevier, New York; 2007:18:1-29.

4. Frasch M: Induction of visceral and cardiac mesoderm by ectodermal Dpp in the early Drosophila embryo. Nature 1995, 374:464-467.

5. Ray RP, Arora K, Nusslein-Volhard C, Gelbart WM: The control of cell fate along the dorsal-ventral axis of the Drosophila embryo. Development 1991, 113:35-54

6. $X u X$, Yin Z, Hudson JB, Ferguson EL, Frasch M: Smad proteins act in combination with synergistic and antagonistic regulators to target Dpp responses to the Drosophila mesoderm. Genes Dev 1998, 12:2354-2370.

7. Yin Z, Frasch M: Regulation and function of tinman during dorsal mesoderm induction and heart specification in Drosophila. Dev Genet 1998, 22:187-200.

8. Yin Z, Xu XL, Frasch M: Regulation of the twist target gene tinman by modular cis-regulatory elements during early mesoderm development. Development 1997, 124:4971-4982.

9. Gajewski K, Zhang Q, Choi CY, Fossett N, Dang A, Kim YH, Kim Y, Schulz RA: Pannier is a transcriptional target and partner of Tinman during Drosophila cardiogenesis. Dev Biol 2001, 233:425-436.

10. Reim I, Frasch M: The Dorsocross T-box genes are key components of the regulatory network controlling early cardiogenesis in Drosophila. Development 2005, 132:4911-4925.

11. Alvarez AD, Shi W, Wilson BA, Skeath JB: pannier and pointedP2 act sequentially to regulate Drosophila heart development. Development 2003, 130:3015-3026.

12. Gajewski K, Fossett N, Molkentin JD, Schulz RA: The zinc finger proteins Pannier and GATA4 function as cardiogenic factors in Drosophila. Development 1999, 126:5679-5688.

13. Jagla T, Bidet Y, Da Ponte JP, Dastugue B, Jagla K: Cross-repressive interactions of identity genes are essential for proper specification of 
cardiac and muscular fates in Drosophila. Development 2002, 129:1037-1047.

14. Klinedinst SL, Bodmer R: Gata factor Pannier is required to establish competence for heart progenitor formation. Development 2003, 130:3027-3038.

15. Lee $H H$, Frasch M: Wingless effects mesoderm patterning and ectoderm segmentation events via induction of its downstream target sloppy paired. Development 2000, 127:5497-5508.

16. Lockwood WK, Bodmer R: The patterns of wingless, decapentaplegic, and tinman position the Drosophila heart. Mech Dev 2002, 114:13-26.

17. Mann T, Bodmer R, Pandur P: The Drosophila homolog of vertebrate Islet1 is a key component in early cardiogenesis. Development 2009, 136:317-326.

18. Park M, Wu X, Golden K, Axelrod JD, Bodmer R: The wingless signaling pathway is directly involved in Drosophila heart development. Dev Biol 1996, 177:104-116.

19. Riechmann V, Irion U, Wilson R, Grosskortenhaus R, Leptin M: Control of cell fates and segmentation in the Drosophila mesoderm. Development 1997, 124:2915-2922.

20. Wu X, Golden K, Bodmer R: Heart development in Drosophila requires the segment polarity gene wingless. Dev Biol 1995, 169:619-628.

21. Newfeld SJ, Takaesu NT: An analysis using the hobo genetic system reveals that combinatorial signaling by the Dpp and Wg pathways regulates dpp expression in leading edge cells of the dorsal ectoderm in Drosophila melanogaster. Genetics 2002, 161:685-692.

22. Knirr S, Frasch M: Molecular integration of inductive and mesodermintrinsic inputs governs even-skipped enhancer activity in a subset of pericardial and dorsal muscle progenitors. Dev Biol 2001, 238:13-26.

23. Johnson AN, Bergman CM, Kreitman M, Newfeld SJ: Embryonic enhancers in the dpp disk region regulate a second round of Dpp signaling from the dorsal ectoderm to the mesoderm that represses Zfh-1 expression in a subset of pericardial cells. Dev Biol 2003, 262:137-151.

24. Johnson AN, Burnett LA, Sellin J, Paululat A, Newfeld SJ: Defective decapentaplegic signaling results in heart overgrowth and reduced cardiac output in Drosophila. Genetics 2007, 176:1609-1624.

25. Affolter $M$, Basler K: The Decapentaplegic morphogen gradient: from pattern formation to growth regulation. Nat Rev Genet 2007, 8:663-674.

26. Sorrentino A, Thakur N, Grimsby S, Marcusson A, von Bulow V, Schuster N, Zhang S, Heldin CH, Landstrom M: The type I TGF-beta receptor engages TRAF6 to activate TAK1 in a receptor kinase-independent manner. Nature cell biology 2008, 10:1199-1207.

27. Xia ZP, Sun L, Chen X, Pineda G, Jiang X, Adhikari A, Zeng W, Chen ZJ: Direct activation of protein kinases by unanchored polyubiquitin chains. Nature 2009, 461:114-119.

28. Geuking P, Narasimamurthy R, Basler K: A genetic screen targeting the tumor necrosis factor/Eiger signaling pathway: identification of Drosophila TAB2 as a functionally conserved component. Genetics 2005 , 171:1683-1694.

29. Takatsu Y, Nakamura M, Stapleton M, Danos MC, Matsumoto K, $\mathrm{O}^{\prime}$ Connor MB, Shibuya H, Ueno N: TAK1 participates in c-Jun N-terminal kinase signaling during Drosophila development. Mol Cell Biol 2000, 20:3015-3026.

30. Adachi-Yamada T, Fujimura-Kamada K, Nishida Y, Matsumoto K: Distortion of proximodistal information causes JNK-dependent apoptosis in Drosophila wing. Nature 1999, 400:166-169.

31. Adachi-Yamada T, O'Connor MB: Morphogenetic apoptosis: a mechanism for correcting discontinuities in morphogen gradients. Dev Biol 2002, 251:74-90.

32. Bier E, Vaessin H, Shepherd S, Lee K, McCall K, Barbel S, Ackerman L, Carretto $R$, Uemura T, Grell E, et al: Searching for pattern and mutation in the Drosophila genome with a P-lacZ vector. Genes Dev 1989, 3:1273-1287.

33. Rebeiz M, Reeves NL, Posakony JW: SCORE: a computational approach to the identification of cis-regulatory modules and target genes in wholegenome sequence data. Site clustering over random expectation. Proc Natl Acad Sci USA 2002, 99:9888-9893.

34. Blackman RK, Sanicola M, Raftery LA, Gillevet T, Gelbart WM: An extensive $3^{\prime}$ cis-regulatory region directs the imaginal disk expression of decapentaplegic, a member of the TGF-beta family in Drosophila. Development 1991, 111:657-666.
35. Byars $C L$, Bates $K L$, Letsou $A$ : The dorsal-open group gene raw is required for restricted DJNK signaling during closure. Development 1999, 126:4913-4923.

36. Hay BA, Wolff T, Rubin GM: Expression of baculovirus P35 prevents cell death in Drosophila. Development 1994, 120:2121-2129.

37. Brand $\mathrm{AH}$, Perrimon N: Targeted gene expression as a means of altering cell fates and generating dominant phenotypes. Development 1993, 118:401-415.

38. Lee YS, Carthew RW: Making a better RNAi vector for Drosophila: use of intron spacers. Methods (San Diego, Calif) 2003, 30:322-329.

39. Spradling AC, Rubin GM: Transposition of cloned $P$ elements into Drosophila germ line chromosomes. Science 1982, 218:341-347.

40. Abrams JM, White K, Fessler LI, Steller H: Programmed cell death during Drosophila embryogenesis. Development 1993, 117:29-43.

41. Booth GE, Kinrade EF, Hidalgo A: Glia maintain follower neuron survival during Drosophila CNS development. Development 2000, 127:237-244.

42. Su MT, Fujioka M, Goto T, Bodmer R: The Drosophila homeobox genes zfh-1 and even-skipped are required for cardiac-specific differentiation of a numb-dependent lineage decision. Development 1999, 126:3241-3251.

43. Cao J, Pellock BJ, White K, Raftery LA: A commercial phospho-Smad antibody detects endogenous BMP signaling in Drosophila tissues. Drosophila Information Services 2006, 89:131-135.

44. Su MT, Golden K, Bodmer R: X-gal staining of Drosophila embryos compatible with antibody staining or in situ hybridization. Biotechniques 1998, 24:918-920, 922.

45. van der Meer J: Optical clean and permanent whole mount preparation for phase-contrast microscopy of cuticular structures of insect larvae. Drosophila Information Services 1977, 52:160-161.

46. Bodmer $\mathrm{R}$ : The gene tinman is required for specification of the heart and visceral muscles in Drosophila. Development 1993, 118:719-729.

47. Han Z, Fujioka M, Su M, Liu M, Jaynes JB, Bodmer R: Transcriptional integration of competence modulated by mutual repression generates cell-type specificity within the cardiogenic mesoderm. Dev Biol 2002, 252:225-240.

48. Jagla K, Frasch M, Jagla T, Dretzen G, Bellard F, Bellard M: ladybird, a new component of the cardiogenic pathway in Drosophila required for diversification of heart precursors. Development 1997, 124:3471-3479.

49. Ward EJ, Skeath JB: Characterization of a novel subset of cardiac cells and their progenitors in the Drosophila embryo. Development 2000, 127:4959-4969.

50. Bates KL, Higley M, Letsou A: Raw mediates antagonism of AP-1 activity in Drosophila. Genetics 2008, 178:1989-2002.

51. Shibuya H, Iwata H, Masuyama N, Gotoh Y, Yamaguchi K, Irie K, Matsumoto K, Nishida E, Ueno N: Role of TAK1 and TAB1 in BMP signaling in early Xenopus development. Embo J 1998, 17:1019-1028.

52. Wu GS: The functional interactions between the p53 and MAPK signaling pathways. Cancer biology \& therapy 2004, 3:156-161.

53. Bryant PJ: Localized cell death caused by mutations in a Drosophila gene coding for a transforming growth factor-beta homolog. Dev Biol 1988, 128:386-395.

54. Umemori M, Habara O, Iwata T, Maeda K, Nishinoue K, Okabe A, Takemura M, Takahashi K, Saigo K, Ueda R, Adachi-Yamada T: RNAiMediated Knockdown Showing Impaired Cell Survival in Drosophila Wing Imaginal Disc. Gene regulation and systems biology 2009, 3:11-20.

55. Yamaguchi K, Shirakabe K, Shibuya H, Irie K, Oishi I, Ueno N, Taniguchi T, Nishida E, Matsumoto K: Identification of a member of the MAPKKK family as a potential mediator of TGF-beta signal transduction. Science 1995, 270:2008-2011.

56. Thakur N, Sorrentino A, Heldin CH, Landstrom M: TGF-beta uses the E3ligase TRAF6 to turn on the kinase TAK1 to kill prostate cancer cells. Future oncology (London, England) 2009, 5:1-3.

57. Kirkpatrick H, Johnson K, Laughon A: Repression of dpp targets by binding of brinker to mad sites. J Biol Chem 2001, 276:18216-18222.

58. Baker JD, McNabb SL, Truman JW: The hormonal coordination of behavior and physiology at adult ecdysis in Drosophila melanogaster. J Exp Biol 1999, 202(Pt 21):3037-3048.

59. Schroder D, Heger J, Piper HM, Euler G: Angiotensin II stimulates apoptosis via TGF-beta1 signaling in ventricular cardiomyocytes of rat. J Mol Med 2006, 84:975-983. 
60. Wencker D, Chandra M, Nguyen K, Miao W, Garantziotis S, Factor SM, Shirani J, Armstrong RC, Kitsis RN: A mechanistic role for cardiac myocyte apoptosis in heart failure. J Clin Invest 2003, 111:1497-1504.

61. Hayakawa Y, Chandra M, Miao W, Shirani J, Brown JH, Dorn GW, Armstrong RC, Kitsis RN: Inhibition of cardiac myocyte apoptosis improves cardiac function and abolishes mortality in the peripartum cardiomyopathy of Galpha(q) transgenic mice. Circulation 2003, 108:3036-3041.

62. Dai RP, Dheen ST, He BP, Tay SS: Differential expression of cytokines in the rat heart in response to sustained volume overload. Eur I Heart Fail 2004, 6:693-703.

63. Takahashi N, Calderone A, Izzo NJ, Maki TM, Marsh JD, Colucci WS: Hypertrophic stimuli induce transforming growth factor-beta 1 expression in rat ventricular myocytes. J Clin Invest 1994, 94:1470-1476.

64. Dorn GW: Apoptotic and non-apoptotic programmed cardiomyocyte death in ventricular remodelling. Cardiovascular research 2009, 81:465-473.

doi:10.1186/1423-0127-18-85

Cite this article as: Yang and Su: Excessive Dpp signaling induces cardial apoptosis through dTAK1 and dJNK during late embryogenesis of Drosophila. Journal of Biomedical Science 2011 18:85.

\section{Submit your next manuscript to BioMed Central} and take full advantage of:

- Convenient online submission

- Thorough peer review

- No space constraints or color figure charges

- Immediate publication on acceptance

- Inclusion in PubMed, CAS, Scopus and Google Scholar

- Research which is freely available for redistribution

Submit your manuscript at www.biomedcentral.com/submit 